\title{
Intrazelluläre Zellzotten in Nahrungsvakuolen von Ciliaten ${ }^{1}$
}

\author{
Gotram Uhlig, Hans Komnick \\ und Karl-Ernst Wohlfarth-Bottermann \\ Biologische Anstalt Helgoland, Meeresstation, Helgoland \\ Zentrallaboratorium für angewandte Übermikroskopie am Zoologischen Institut \\ der Universität Bonn
}

\begin{abstract}
Lamellar microvilli in food vacuoles of ciliates. With progressing age, the food vacuoles of Metafolliculina adreresi develop a border of folds, which attains its most pronounced differentiation at a stage where maximum resorption of nutrient substances presumably takes place. This border of folds, similar to the well known brush border at the free cell surface, consists of lamellar cytoplasm protuberances and causes a considerable enlargement of the membrane area of the food vacuole within the cell, thus favoring the permeation of nutrient substances. In old food vacuoles the border of folds undergoes involution, an observation that also points to the functional significance of this structure. In Metafolliculina the degree of digestion of the vacuole contents and the morphological changes of the vacuole wall enable a distinction to be made between 4 different stages of age and function of the food vacuoles respectively: (1) ingestion vacuoles, (2) digestion vacuoles, (3) resorption vacuoles and (4) egestion vacuoles. The resorption vacuole evidently shows structural and functional analogies to the resorbing intestinal epithelia of Metazoa. The morphological findings are discussed in the light of current concepts on substance uptake by the cell.
\end{abstract}

\section{EINLEITUNG}

Zellzotten oder Mikrozotten sind kleine Cytoplasmaprotuberanzen, die an der freien Oberfläche vieler Zellen beobachtet werden können (vgl. KомNıcK \& WoHLPARTH-BOTTERMANN 1964). Nach dem Erscheinungsbild haben KOMNICK \& WoHLFARTH-BOTTERMANN (1964) zwei extreme Formen unterschieden: fingerförmige Zellfortsätze als Mikrovilli (Policaro et al. 1955) und kammförmige Zellfortsätze als Mikrosinus (Moericke \& Wohlfarth-Bottermann 1960). Zwischen diesen beiden Grundformen gibt es zahlreiche, mehr oder weniger polymorphe Übergangsformen. Während ideale Mikrosinus bislang nur recht selten und unseres Wissens bisher nur bei Insekten gefunden wurden (Moericke \& Wohlfarth-Bottermann 1960; SMIth \& Littau 1960; Steinbrecht 1964; Steinbrecht \& Schneider 1964), sind ideale Mikrovilli offenbar weit verbreitet, so daß diese Bezeichnung sich generell für Zellzotten eingebürgert hat.

1 Mit Unterstützung der Deutschen Forschungsgemeinschaft. 
An der apikalen Oberfläche von Epithelzellen mit vorwiegend resorptiver Funktion und hoher Resorptionsleistung - als bekannteste und eindrudksvollste Beispiele seien hier nur das Dünndarmepithel (vgl. unter anderem Zetrerquist 1956, PaLAy \& Karlin 1959a, b, Ruska 1960, Bergener 1962 und Sedar 1964) und das Epithel der proximalen Nierentubuli (vgl. unter anderem RHodin 1954, 1958, KurTz 1964, Thoenes 1961, 1965 und Sitte 1965) von Wirbeltieren erwähnt - sind Mikrovilli besonders zahlreich anzutreffen und treten zu einem sogenannten Bürstensaum zusammen, der die resorbierende Zelloberfläche um ein Vielfaches vergrößert (vgl. unter anderem Komnick \& Wohlfarth-Bottermann 1964, Thoenes 1965). Aus der häufig beobachteten Koinzidenz von resorptiver Funktion und Mikrozottenbesatz wurde daher auf eine resorptive Funktion der Mikrozotten selbst geschlossen und das Auftreten beziehungsweise Fehlen von Zellzotten wiederum als Hinweis auf das Vorhandensein respektive Fehlen einer resorptiven Funktion von Zellen gewertet (vgl. unter anderem BAUTZMANN \& SChMidT 1960, KomNick 1965).

Um so erstaunlicher ist es, daß bei Nahrungsvakuolen von Protozoen, bei denen - zumindest in bestimmten Stadien - eine hohe Resorptionsleistung der Vakuolenwand vermutet werden muß, bislang keine entsprechenden resorptiven Oberflächenstrukturen beschrieben wurden. Ober solche Differenzierungen der Vakuolenwand wird in der vorliegenden Mitteilung berichtet. Dieser Fall ist auch insofern von besonderem morphologischem und funktionellem Interesse, als hier nicht wie üblich Zellzotten als Differenzierung der freien Zelloberfläche in ein "äußeres Milieu“ ragen, aus dem sie bestimmte Stoffe entnehmen (Permeation) und in die Zelle transportieren, sondern auch noch (beziehungsweise erst) ausgebildet werden, wenn dieses ursprünglich äußere Milieu oder bestimmte Anteile daraus bereits durch andere Mechanismen der Stoffaufnahme (Phagozy tose) in die Zelle inkorporiert worden sind.

\section{MATERIAL UND METHODE}

Die Untersuchung wurde an den Nahrungsvakuolen des sessilen, gehäusebauenden Flaschentierchens Metafolliculina andrewsi HaDžI (Andrews 1923, HaDžr 1951) durchgeführt, einem heterotrichen Ciliat, das aus dem Litoral um Helgoland stammt, seit etwa drei Jahren in Klonkulturen in der Biologischen Anstalt Helgoland gehalten und mit Dunaliella und Cryptomonas (aus nicht sterilen Kulturen) gefüttert wird (Uhlig 1963a, b und 1965). Die auf dem Boden der Kulturschale festsitzenden, adulten Tiere wurden zur Fixation mit einer 1\% wasser (deren $\mathrm{pH}$ mit 2,5 n KOH auf 7,2 eingestellt war) übergossen, während der Entwässerung in Aceton in 0,5\% Uranylacetat und 1\% Phosphorwolframsäure nach WoHLfarth-BOtTERMANN (1957) in toto nachkontrastiert, in Vestopal W (Firma Jaeger, Vésenaz, Schweiz) eingebettet und auf einem Ultrotome der Firma LKB (Stockholm) geschnitten. Die Schnitte wurden mit einem Elektronenmikroskop Typ UM 100 d der Firma Siemens (Berlin) untersucht ${ }^{2}$.

2 Für technische Assistenz sind wir Frau B. Koeppen-Lesche und Frau M. Sauernheimer zu Dank veroflichtet. 


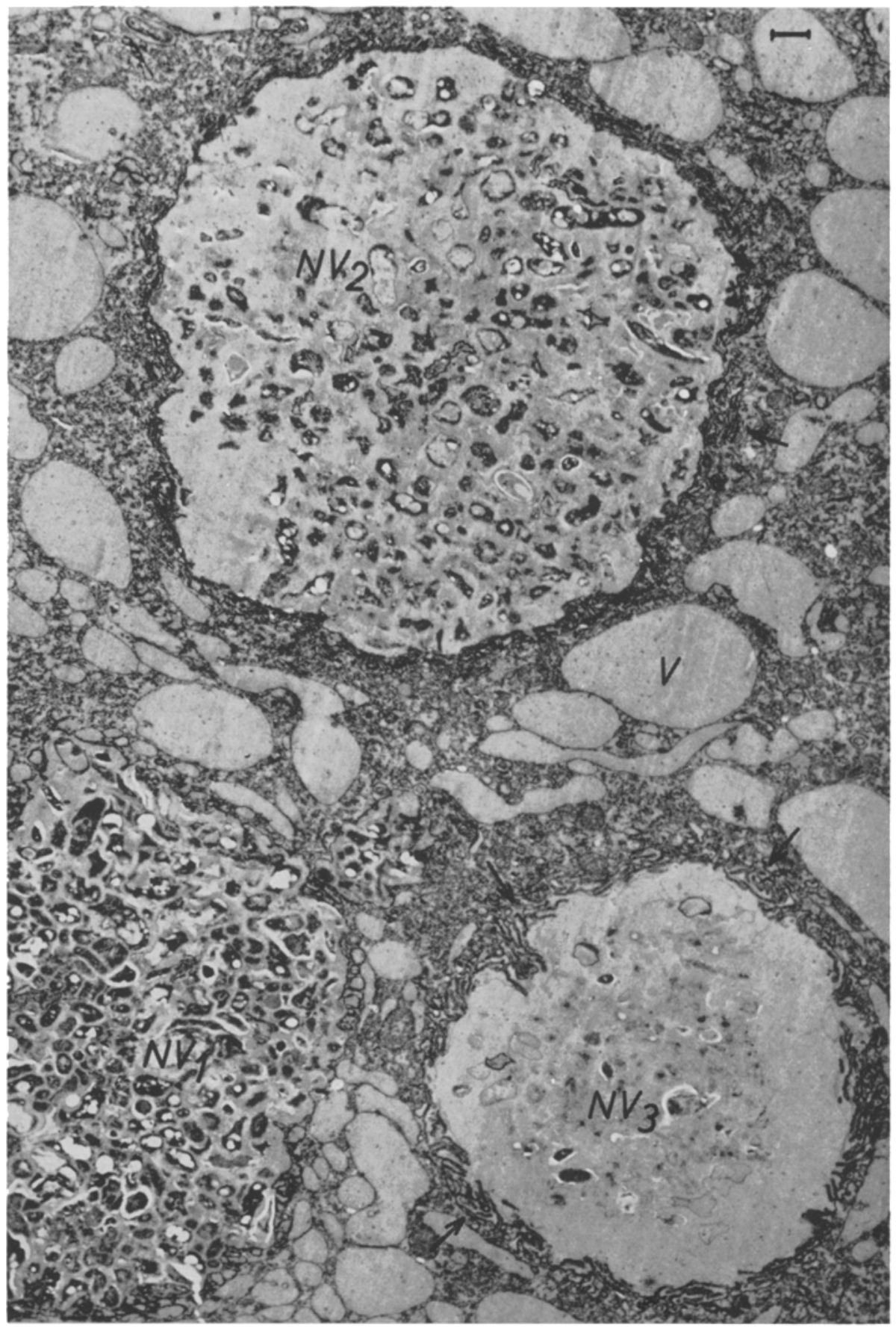

Abb. 1: Metafolliculina andrewsi. Ausschnitt aus dem zentralen Cytoplasma mit 3 Nahrungsvakuolen verschiedenen Alters. $N V_{1}$ jüngere Nahrungsvakuole; $N V_{2}$ und $N V_{3}$ Nahrungsvakuolen mittleren Alters. Bei $N V_{2}$ sind die Mikrozotten der Vakuolenwand noch schwach, bei $N V_{3}$ bereits stärker entwickelt (Pfeilmarkierungen), $V$ Vakuolen, die normalerweise das zentrale Cytoplasma dieser Tiere durchsetzen. Vergrößerung 4500:1 


\section{BEFUNDE}

Das genaue Alter der Nahrungsvakuolen von Metafolliculina andrewsi läßt sich in Dünnschnittbildern nicht bestimmen, da die Vakuolen nach ihrer Abschnürung vom Cytopharynx durch die Cyclose im Zelleib umhergetragen werden. Diese Cyclose erfolgt nicht in festgelegten Bahnen, so daß die Nahrungsvakuolen unregelmäßig verteilt werden. Ihre Lage an einer bestimmten Stelle des Zellkörpers läßt daher keine Rückschlüsse auf das Alter zu. Lediglich an Hand des Verdauungsgrades der aufgenommenen Nahrungsorganismen kann das rela tive Alter der Vakuolen ungefähr bestimmt werden (vgl. hierzu auch SchneIder 1964), das heißt, bei einer Beurteilung zweier

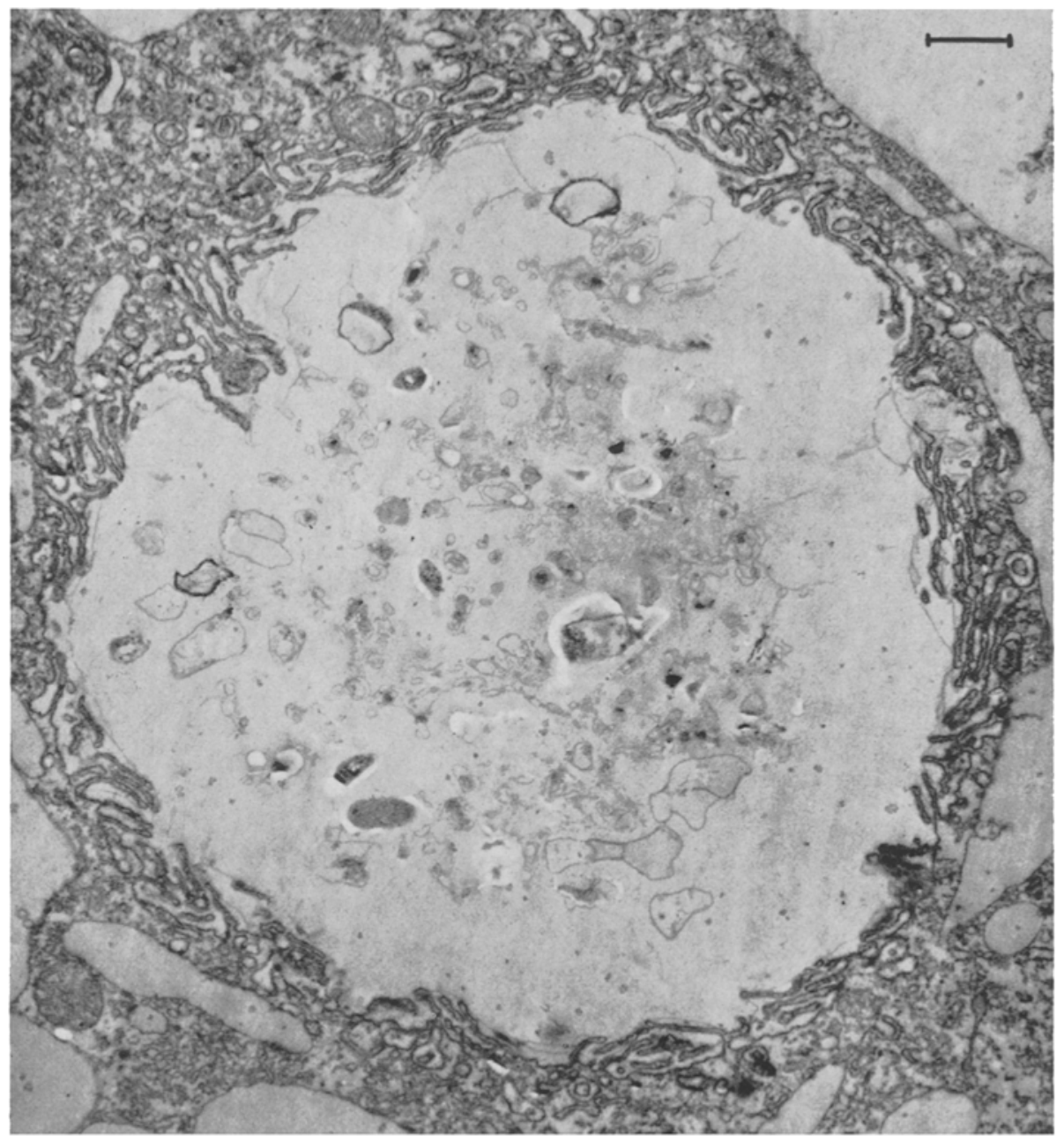

Abb. 2: Metafolliculina andrewsi. Nahrungsvakuole mittleren Alters ( $N V_{3}$ aus Abb. 1 stärker vergrößert) mit vorwiegend zirliulär angeordneten Mikrozotten. Vergrößerung 10 500:1 


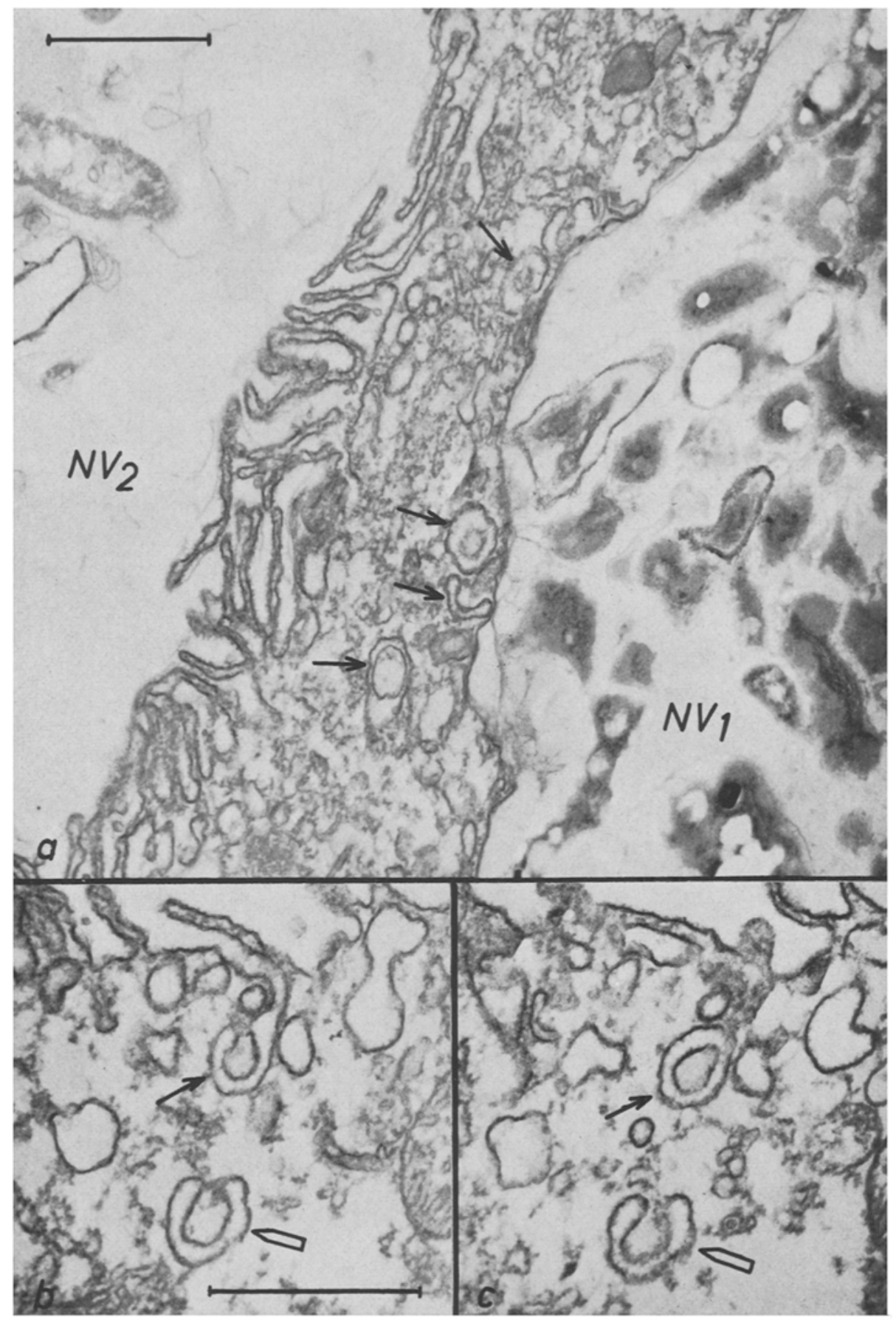

Abb. 3: Metafolliculina andrewsi. a Ausschnitt aus einer jungen Nahrungsvakuole mit glatter Wand $\left(N V_{1}\right)$ und einer älteren Nahrungsvakuole mit Mikrozotten $\left(N V_{2}\right)$. Die Pfeilmarkierungen weisen auf hufeisenförmige und konzentrische Membranprofile. Vergrößserung 22 000:1. $b$ und $c$ Ausschnitte aus einer ältcren Nahrungsvakuole. Ein hufeisenförmiges Membranprofil steht mit dem Vakuolenlumen in Verbindung $(b)$; im Folgeschnitt $(c)$ erscheint ein ,isolierter Doppelnembranring" (einfache Pfeile). Die Doppelpfeile deuten auf eine tieferliegende gleiche Struktur. Vergrößerungen 29000:1 
oder mehrerer Vakuolen kann nur gesagt werden, welche Vakuole älter und welche jünger ist. Dieses Kriterium erlaubt zum Beispiel über die drei in Abbildung 1 dargestellten Nahrungvakuolen die Feststellung: $N V_{3}$ älter als $N V_{2}$ älter als $N V_{1} \cdot \mathrm{Zu}$ einer genauen Altersbestimmung wären systematische, vitalmikroskopische (ANDREWs 1946) und elektronenmikroskopische Untersuchungen erforderlich, um schließlich eine bestimmte Nahrungsvakuole allein an Hand des Verdauungsgrades der Beute in eine mehr oder weniger enge Altersspanne einordnen zu können. Da der Zweck dieser Untersuchung nicht eine umfassende Darstellung des Schicksals der Nahrungsvakuolen von der Ingestion bis zur Egestion ist, sondern lediglich die Beschreibung bestimmter morphologischer - wenn auch zeitlich aufeinanderfolgender - Veränderungen der Vakuolenwand (die als Nebenbefunde von Untersuchungen mit anderem Ziel erhoben wurden), genügt in diesem Falle eine relative Altersbestimmung.

\section{Die jüngeren Nahrungsvakuolen}

Jüngere Nahrungsvakuolen (Abb. 1, NV ; Abb. 3, NV $\mathrm{NV}_{1}$ Abb. 5a) sind in der Regel dicht mit Beuteorganismen (vorwiegend Bakterien) gefüllt, die schon mehr oder minder deutliche Anzeichen lytischer Veränderungen erkennen lassen. Anzeichen einer Oberflächenvergrößerung der Vakuolenwand durch Faltenbildungen sind nicht mit Sicherheit zu erkennen; abgesehen von gelegentlichen Unregelmäßigkeiten, die teilweise vitale Verformungen sein mögen (ANDREws 1946), teilweise aber auch auf präparativ bedingte Schrumpfungen zurückgeführt werden könnten, erscheint die Vakuolenmembran relativ glatt. Dies gilt insbesondere für ganz junge Nahrungsvakuolen. Bei etwas älteren Nahrungsvakuolen zeigt das die Vakuole unmittelbar umgebende Cytoplasma zahlreiche vakuoläre und tubuläre Schnittprofile. Die naheliegende Frage, ob es sich bei den kleinen Vakuolen, die auch um junge Nahrungsvakuolen von Paramecium auftreten (JuRAND 1961, SchNEIDER 1964) um Enzymvehikel handelt, die die frisch gebildete Nahrungsvakuole mit Verdauungsenzymen versorgen (vgl. MüLLER \& Törö 1962, Müller, RöHlich, TóTH \& TöRö 1963) beziehungsweise um mikropinocytotische Sekundärvesikel (wie bei den Nahrungsvakuolen von Peritrichen), in denen Anteile des Hauptvakuoleninhaltes verdaut werden (vgl. Carasso et al. 1964, Favard \& Carasso 1964), wäre erst nach einer histochemischen Prüfung zu beantworten. Eine strukturelle Parallele zu den Befunden an Peritrichen sind die auffälligen hufeisenförmigen Schnittprofile ("cupules“) und konzentrischen "Doppelmembranringe“. Nach Carasso et al. (1964) und Favard \& Carasso (1964) handelt es sich bei Peritrichen dabei um von der Nahrungsvakuole abgeschnürte Pinocytosevesikel, die im Verlaufe von Resorptionsprozessen abgeplattet und gekrümmt und schließlich wieder in die Nahrungsvakuole abgegeben werden. Es erscheint uns zweifelhaft, ob trotz der strukturellen Ähnlichkeit auch eine funktionelle Parallele besteht. Eine genaue Strukturanalyse bestärkt uns in der Ansicht, daß es sich bei Metafolliculina (wo diese Gebilde besonders zahlreich um junge Nahrungsvakuolen auftreten, aber auch vereinzelt um Nahrungsvakuolen jeden Alters anzutreffen sind) dabei um Strukturen handelt, die durch zweimalige Faltung der Nahrungsvakuolenmembran zustande kommen: Die Nahrungsvakuole schiebt eine mehr oder weniger tiefe Tasche in das Cyto- 


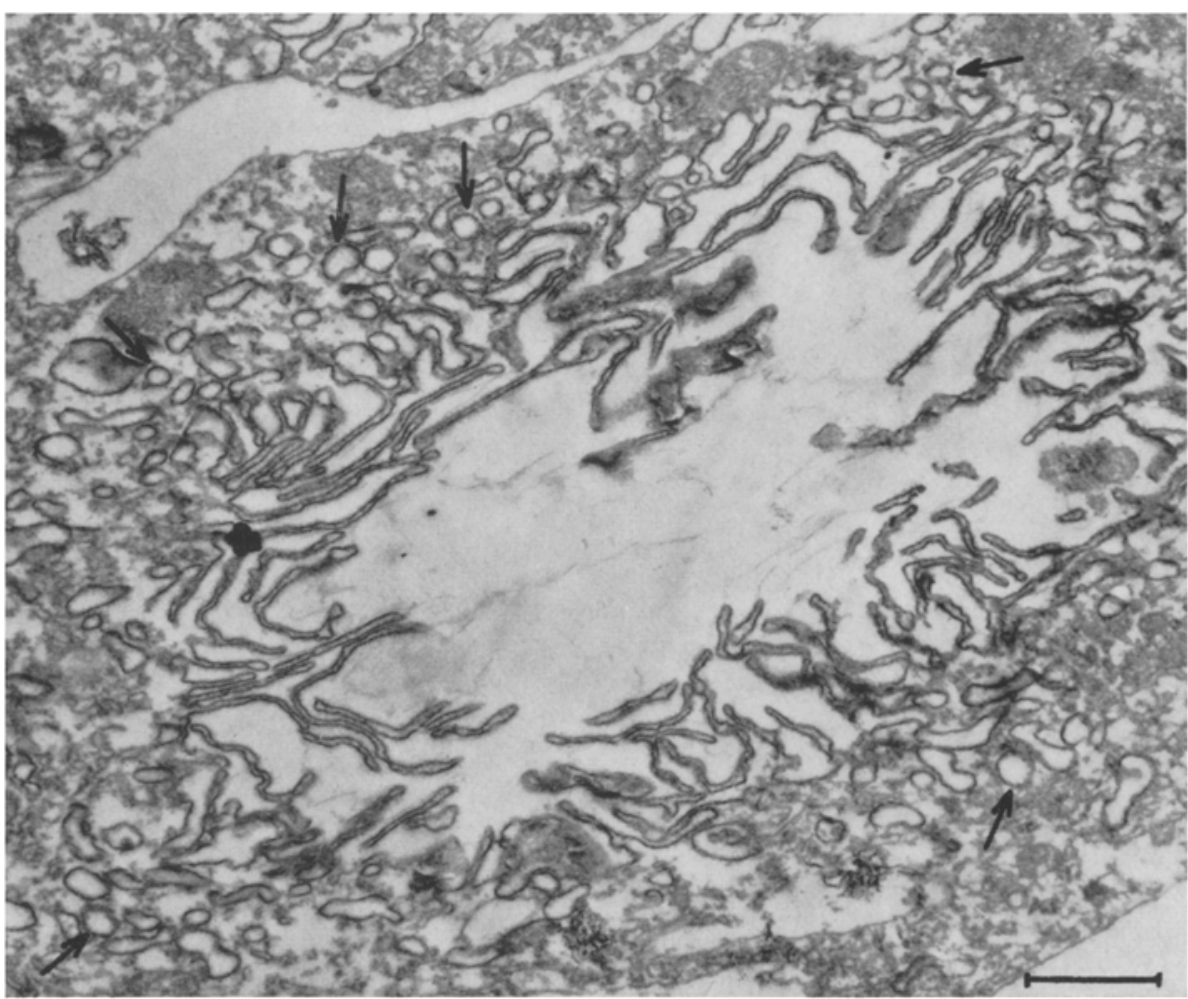

Abb. 4: Metafolliculina andrerosi. Altere Nahrungsvakuole mit wohlausgebildetem Faltensaum und zahlreichen Sekundärvesikeln (Pfeile). Vergrößerung 14000:1

plasma vor, in die dann wiederum eine Cytoplasmaprotuberanz eingesenkt wird, so daß dann je nach Schnittrichtung hufeisenförmige Profile oder zwei konzentrische Membranringe erscheinen (Abb. $3 b$ und $c$ ). Statt eines inneren Membranringes findet man um junge Nahrungsvakuolen auch häufig ein oder mehrere „tubuläre" Profile, die stellenweise mit dem übrigen Cytoplasma in Verbindung stehen. Es ist daher nicht der Verdacht von der Hand zu weisen, daß es sich dabei um Vorstadien der später zu beschreibenden Mikrozotten handelt. Triff diese Interpretation $\mathrm{za}$, dann würden die Mikrozottenbildungen zunächst am Grunde kryptenartiger Aussackungen der Nahrungsvakuolen beginnen, möglicherweise weil sich diese Protuberanzen in der Vakuole selbst aus Raummangel infolge des dichtgepackten Vakuoleninhaltes noch nicht entfalten können.

Die Nahrungsvakuolen mittleren Alters

Nahrungsvakuolen mittleren Alters (Abb. 1, $\mathrm{NV}_{2}$ und $\mathrm{NV}_{3}, \mathrm{Abb} .2$ und $5 \mathrm{~b}$ ) sind durch die weiter fortgeschrittene Lysis der Nahrungsorganismen gekennzeichnet, deren 


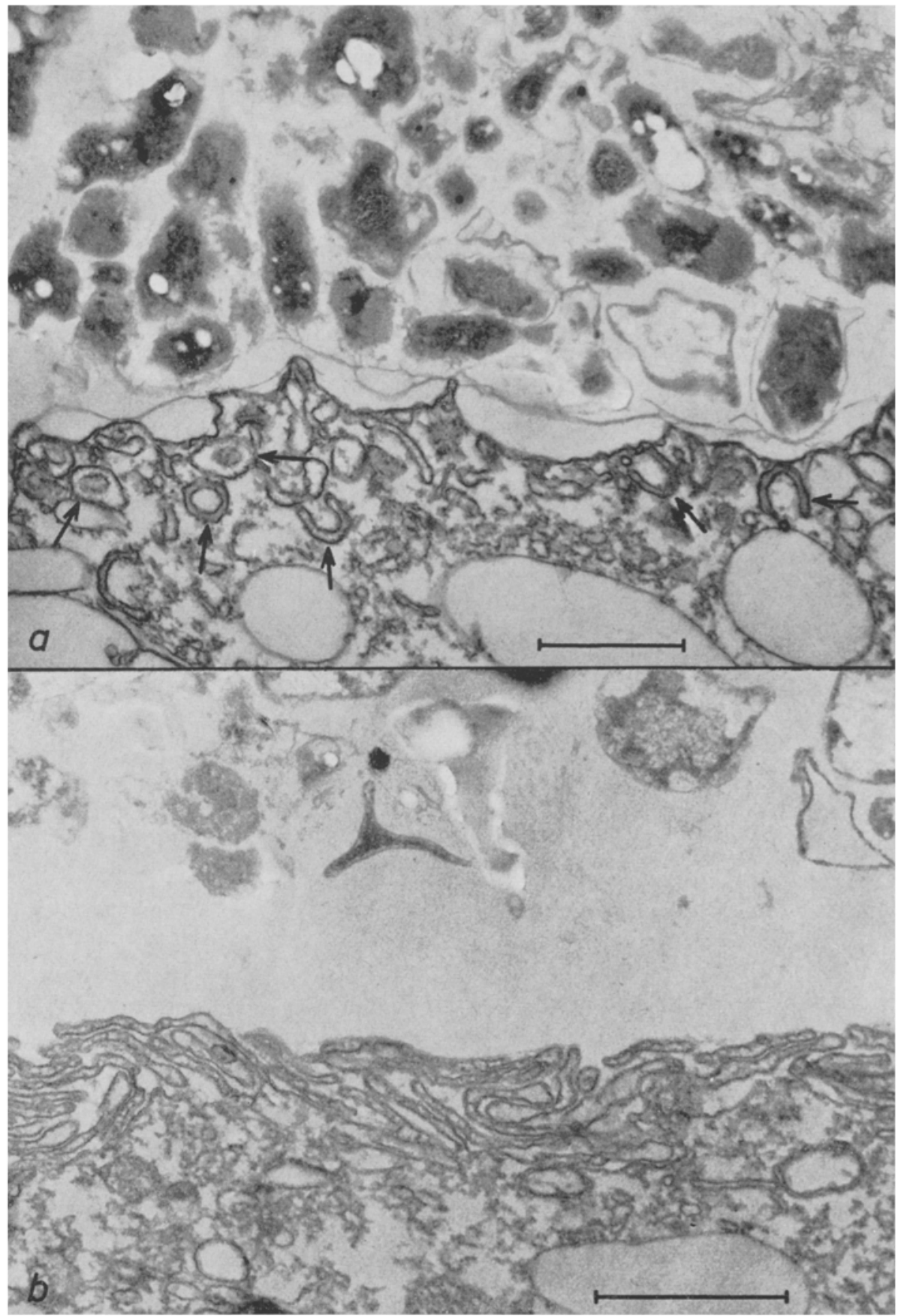

Abb. 5: Metafolliculina andrezesi. a Ausschnitt aus einer jüngeren Nahrungsvakuole ohne Mikrozotten (Ingestionsvakuole). Die Pfeile deuten auf hufeisenförmige und konzentrische Membranprofile. Vergrößerung 19000:1. $b$ Ausschnitt aus einer Nahrungsvakuole mittleren Alters mit zirkulär verlaufenden Mikrozotten (Digestionsvakuole). Vergrößerung 25 500:1 

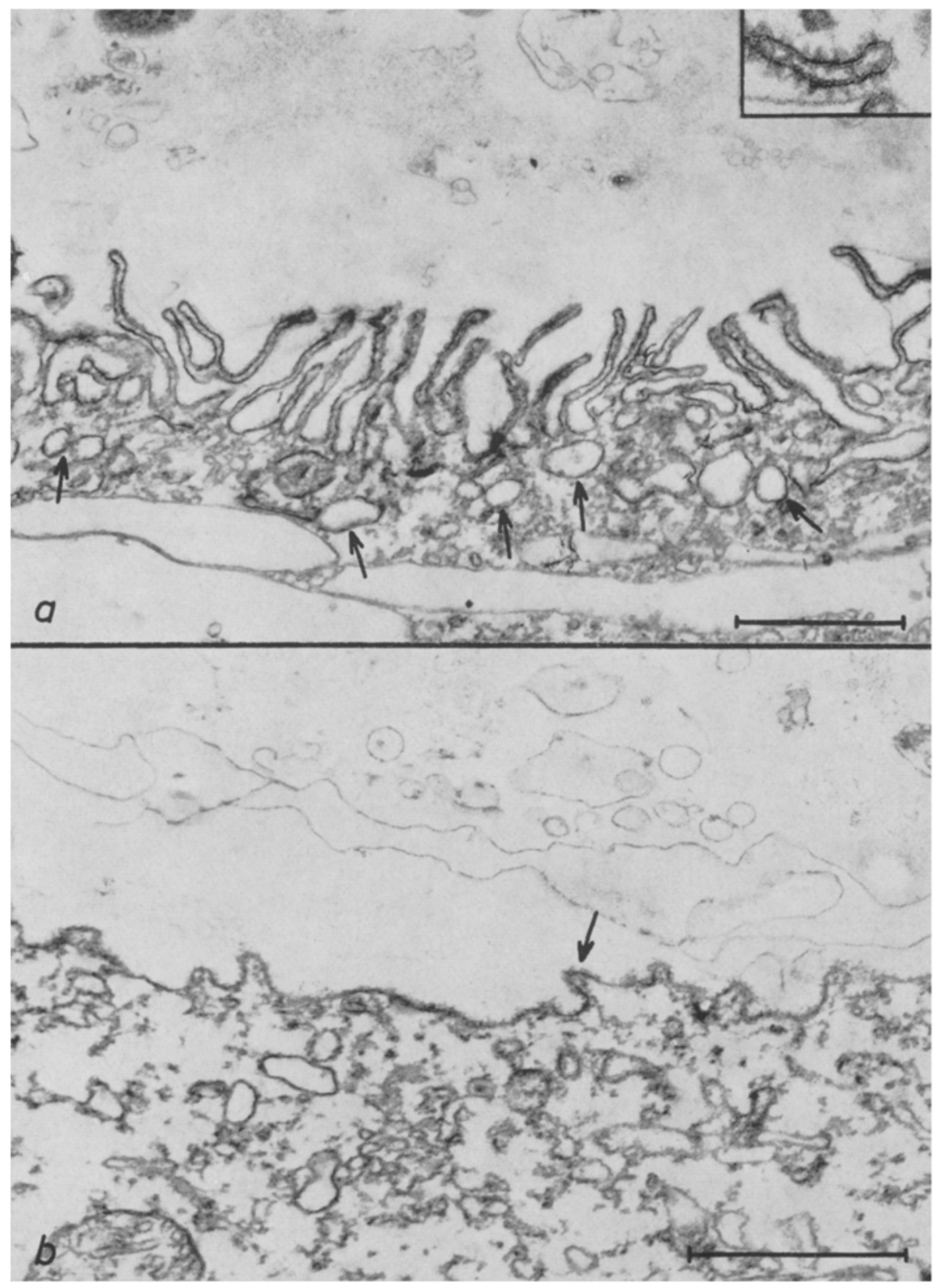

Abb, 6: Metafolliculina andrewsi. a Ausschnitt aus einer älteren Nahrungsvakuole mit radiär stehenden Mikrozotten (Resorptionsvakuole) und Sekundärvesikeln (Pfeile). Beachte die härchenförmig organisierte Deckschicht der Mikrozottenmembran oben rechts! Vergrößerung $21000: 1$. Bildeinsatz $64000: 1$. $b$ Ausschnitt aus einer ganz alten Nahrungsvakuole mit reduzierten Mikrozotten (Egestionsvakuole). Die Pfeilmarkierung deutet auf die Deckschicht der Vakuolenmembran. Vergrößerung 28 000:1 
dichte Packung allmählich aufgelockert wird. Die Vakuolen werden in diesem Stadium von einer Fülle tubulärer Schnittprofile umlagert, die sich in ihrer Gesamtheit bei genauerer Betrachtung und bei stärkerer Vergrößerung als hochgradige Faltenbildung der Vakuolenmembran entpuppen. Diese Falten erscheinen weitgehend ungeordnet; sie biegen zumeist an ihrer Wurzel um und scheinen sich dem Vakuoleninhalt anzuschmiegen, das heißt sie verlaufen in bezug auf die Vakuole vorwiegend zirkulär (Abb. 5b). Mit zunehmendem Alter der Nahrungsvakuole richten sie sich dann auf (Abb. 3a, NV). Diese Oberflächendifferenzierung der Vakuolenmembran mit zunehmendem Alter ist besonders eindrucksvoll in solchen Bildern zu erkennen, in denen Nahrungsvakuolen jungen und mittleren Alters dicht nebeneinanderliegen (Abb. 3a).

Die Beobachtung, daß die Falten stellenweise noch in relativ weiter Entfernung von der "Vakuolenoberfläche" anzutreffen sind, scheint zugunsten der oben angeführten Interpretation zu sprechen, wonach die Faltenbildung zunächst in taschenartigen Krypten der Vakuole auftreten. Das Lumen dieser kleinen Krypten kann oftmals sekundär durch Falten fast völlig wieder ausgefüllt sein.

\section{Die älteren Nahrungsvakuolen}

Bei älteren Nahrungsvakuolen (Abb. 4 und 6a) sind die Nahrungsorganismen schon weitgehend verflïssigt. Im Lumen der Vakuole finden sich nur noch vereinzelte Membranreste und mitunter Konglomerate von unverdautem Material sowie diffuse wolken- oder flockenartige Areale, die sich auf Grund ihrer etwas größeren Elektronendichte vom hellen Untergrund abheben und die wahrscheinlich ausgefällte, fixierbare Anteile des flüssigen Vakuoleninhaltes darstellen. Die Wand dieser Vakuolen zeigt eine äußerst unregelmäßige Kontur und ist durch Faltenbildung beziehungsweise Cytoplasmaprotuberanzen extrem zerklüftet. Die Falten stehen jetzt mehr oder weniger senkrecht zur Vakuolenzirkumferenz, also radiär in bezug auf die Vakuole, und tauchen weit in den Vakuoleninhalt hinein. Diese intrazellulären Mikrozotten bilden einen dichten Faltensaum und erwecken in Abbildung 6a fast den Eindruck eines wenig geordneten Bürstensaumes. Da trotz ihrer geringen Ordnung runde Querschnittsprofile nur ganz selten angetroffen werden, handelt es sich aber vorwiegend um faltenförmige, mikrosinusartige Zotten, die vielerorts verzweigt sind.

Die Membran der Nahrungsvakuole und die der Mikrozotten trägt, wie in der Ausschnittsvergrößerung in Abbildung $6 \mathrm{a}$ deutlich zu erkennen ist, auf der an den Vakuoleninhalt grenzenden Seite eine diffuse Deckschicht, die „härchenförmig“ organisiert zu sein scheint und an die Mucoidschicht der Zellmembran von Amöben erinnert (vgl. unter anderem Komnick \& WoHLFARTH-BotTERMAnN 1965). Obwohl sich nicht gänzlich die Möglichkeit ausschließen läßt, daß es sich bei dieser Deckschicht um an der Vakuolenmembran während der Fixation niedergeschlagenes Material des flüssigen Vakuoleninhaltes handelt, spricht ihr Vorhandensein auch in jüngeren Nahrungsvakuolen, bei denen die Lysis der Nahrung noch nicht so weit fortgeschritten ist, gegen diese Annahme.

In unmittelbarer Nähe der Nahrungsvakuole liegen zahlreiche kleinere "Sekundärvesikel“ (Abb. 4 und 6a), die auch um die älteren Nahrungsvakuolen von Para- 

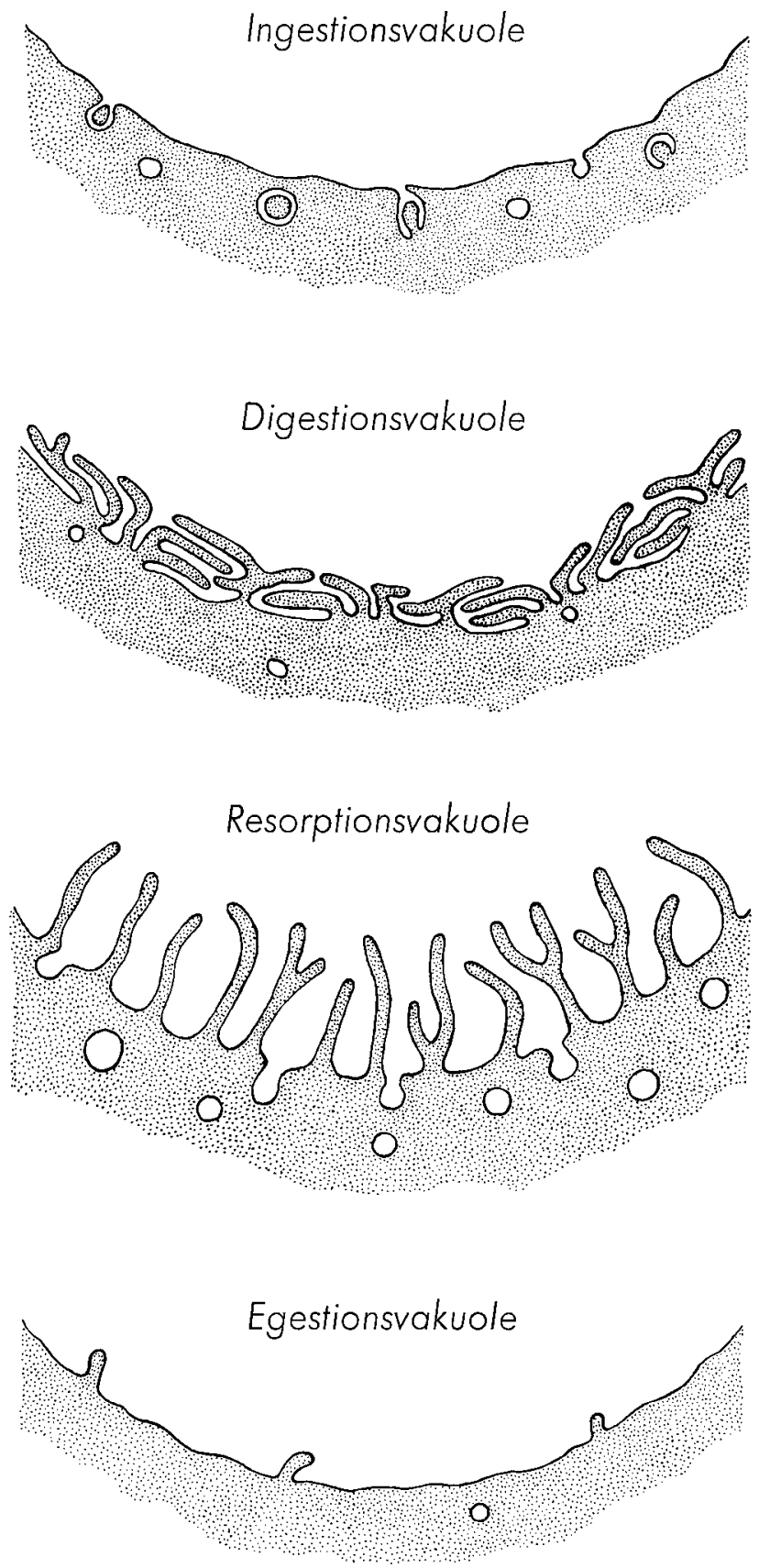

Abb. 7. Schematische Darstellung der strukturellen Veränderungen der Nahrungsvakuolenwand von Metafolliculina andrewsi im Verlaufe des Verdauungsprozesses 
mecium (SCHNEIDER 1964) anzutreffen sind. Vermutlich handelt es sich dabei um Einstülpungen und Abschnürungen der Nahrungsvakuolenmembran, worauf die Deckschicht ihrer Hüllmembran hinzudeuten scheint.

\section{Die ganz alten Nahrungsvakuolen}

Ganz alte Nahrungsvakuolen (Abb. 6b) unterscheiden sich hinsichtlich ihres Inhaltes meistens kaum von den zuvor beschriebenen älteren Vakuolen. In den Fällen, in denen sie nicht durch ihre Lage in unmittelbarer Nähe der Cytopyge zu bestimmen sind, können sie jedoch leicht durch die Reduktion der Falten erkannt werden. Diese Reduktion führt schließlich zum fast völligen Verschwinden der Mikrozotten; nur hin und wieder sind noch kleine Cytoplasmaprotuberanzen anzutreffen. Die Vakuolenmembran trägt auch noch in diesem Altersstadium eine Deckschicht (Abb, 6b).

In den Abbildungen 5 und 6 sind die für die vier besprochenen Altersstadien charakteristischen Strukturaspekte der Nahrungsvakuolenwand von Metafolliculina andrewsi zusammengestellt und in Abbildung 7 schematisch verdeutlicht. Wenn wir auf Grund der morphologischen Veränderung des Vakuoleninhaltes und der Vakuolenwand diese Altersstadien als Ingestions-, Digestions-, Resorptions- und Egestionsvakuole bezeichnen, so sind wir uns darüber im klaren, daß wir damit mehr oder weniger willkürliche Cäsuren in den kontinuierlichen Prozeß der intrazellulären Verdauung setzen.

\section{DISKUSSION}

Die Beobachtung von intrazellulären Mikrozotten in den Nahrungsvakuolen von Metafolliculina andrewsi ist aus cytomorphologischer Sicht - wie bereits einleitend erwähnt - insofern ungewöhnlich, als solche Strukturen bisher nur als Differenzierungen der freien Zelloberfläche bekannt sind und normalerweise in ein extrazelluläres Milieu hineinragen. Zwar existieren auch zahlreiche Mitteilungen über Mikrozotten in sogenannten „intrazellulären Sekretkanälchen" (Literatur bei KомNICK \& WOHLFARTH-BOTTERMANN 1964), doch handelt es sich bei diesen Kanälchen in Wirklichkeit um extrazelluläre Räume, die mit der Drüsenlichtung in offener Verbindung stehen. Das bekannteste Beispiel hierfür sind die Korbkapillaren von Belegzellen ${ }^{3}$ (vgl. unter anderem Helander 1962, 1965). Der Inhalt der Nahrungsvakuolen von Metafolliculina war zwar ursprünglich ebenfalls extrazelluläres Milieu, die Mikrozotten werden hier jedoch erst relativ spät ausgebildet, nachdem die Nahrungsvakuole in die Zelle aufgenommen wurde und somit eine intrazelluläre Vakuole ist.

Aus zellphysiologischer Sicht ist gerade dieses späte Auftreten der Mikrozotten in den Nahrungsvakuolen von Interesse, das heißt zu einem Zeitpunkt, wo die Lysis der

${ }^{3}$ Bei den Mikrovilli der Korbkapillaren yon Belegzellen ist es außerdem fraglich, ob ihnen eine resorptive Funktion zugeschrieben werden kann (КомNICK 1963), da sie die sezernierende Oberfläche dieser Zelle vergrößern (zu dieser Frage vgl. auch Komnick \& WoHLFARTH-BOTTERMANN 1964) und da ihnen nach bisherigen Beobachtungen die äußere Deckschicht fehlt (HeLander 1965). 
Beuteorganismen schon fortgeschritten und wo daher die Resorption der Nahrung zu vermuten ist. Dieser Befund hat eine Parallele in den Beobachtungen von Gresson $8 x$ Threadgold (1959) an den Darmzellen von Fasciola bepatica, wo Mikrozotten („absorptive processes“) erst ausgebildet werden, wenn sich der Darm mit Nahrung füllt.

Bei der zellulären Stoffaufnahme werden heute allgemein zwei Mechanismen unterschieden (vgl. Schmidt 1965): Die Permeation einerseits, die durch die Permeabilitäts- und Transporteigenschaften der Zellmembran bestimmt wird, und die Endocytose andererseits, die Phagocytose, Pinocytose und eine Reihe ähnlicher Vorgänge umfaßt (vgl. hierzu das Symposion über Lysosomen 1963, p. 126 und 411-412 sowie Holter 1965). Das Charakteristikum der endocytotischen Stoffaufnahme „ist die Verlegung der Membranbarriere von der Oberfläche in das Zellinnere" (Holter 1965). In diesem Sinne muß es heute als fraglich erscheinen, ob die Endocytose letztlich als alternativer Mechanismus zur Permeation aufgefaßt werden kann, da sich im Anschluß an die endocytotische Stoffaufnahme erneut die Frage nach der Permeation - jetzt Permeation der Vakuolenmembran - stellt. Diese Frage hat Holter (1965) eingehend erörtert und folgende drei Möglichkeiten diskutiert.

1. Die invaginierte Zellmembran, das heißt die Hüllmembran der Vakuolen, könnte innerhalb der Zelle gewisse Veränderungen zugunsten einer Permeabilitätserhöhung erfahren. Der Extremfall wäre die partielle Fragmentierung oder vollständige Auflösung der Vakuolenmembran und damit die Freilegung des Vakuoleninhalts im Grundplasma. Die letzte Möglichkeit ist trotz einiger morphologischer Anzeichen (vgl. unter anderem WoHLfartH-BotTERmann 1960) wegen der Artefaktgefahr heute umstritten.

2. Zahlreiche Befunde deuten darauf hin, daß die Stoffentnahme aus der Endocytose-Vakuole durch mikropinocytotische Vesikel erfolgt (vgl. unter anderem JuRand 1961, Müller \& Röhlich 1961, Schneider 1964, Roth 1960, ChapmanAndresen \& Nilsson 1960, Carasso et al. 1964, Favard \& Carasso 1963, 1964). Diese Möglichkeit, die auch in unseren Bildern angedeutet wird (Abb, 4 und 6a), bleibt letztlich jedoch unbefriedigend, da sich jetzt zum zweiten Male die Frage nach der Permeation der Sekundärvesikelmembran stellt. Es muß also erneut das unter Punkt 1 genannte Postulat erhoben oder angenommen werden, daß die Sekundärvesikel mit anderen Zellorganellen verschmelzen (vgl. STaubesand 1965). So hat denn Rотн (1960) diese sekundäre Mikropinocytose als eine Oberflächenvergrößerung der Hauptvakuole zugunsten einer erhöhten Permeation gedeutet (vgl. hierzu jedoch auch HaYw ARD 1963).

3. Im Sinne einer Oberflächenvergrößerung der Vakuolenmembran müssen auch die von Brandt \& Pappas (1962) und HaYward (1963) beschriebenen Verzweigungen der Pinocytose-Vakuolen von Amöben aufgefaßt werden, die ältere Vakuolen in ein beinahe labyrinthartiges Hohlraumsystem umwandelten. Diese Oberflächenvergrößerung dürfte ebenfalls die Permeation begünstigen.

Die Permeation kann durch eine Steigerung der Membranpermeabilität (Punkt 1) oder durch eine Vergrößerung der Membranfläche (Punkt 3) begünstigt werden. Bei der unter Punkt 2 angeführten Sekundärvesikulation könnten beide Faktoren zugleich eine Rolle spielen. Solange eine partielle oder totale Auflösung der Vakuolenmembran 
nicht zweifelsfrei bewiesen ist, bleibt die Permeation ein entscheidendes Kriterium auch der endocytotischen Stoffaufnahme. Holter (1965) betrachtet in diesem Zusammenhang "die Pinocytose als Hilfsmechanismus des Membrantransports ..., welcher durch die Translokation großer Anteile der Zelloberfläche ins Zellinnere und darauf folgende Membranumwandlungen die Transportprozesse in ein anderes zelluläres Niveau verlegt, aber die entscheidenden physiologischen Merkmale des Membrantransports unverändert läßt ..." (p. 138-139).

Die Mikrozotten in den älteren Nahrungsvakuolen von Metafolliculina sind unserer Ansicht nach als Oberflächenvergrößerung der Nahrungsvakuolenmembran zugunsten der Permeation von Nährstoffen zu deuten. Am Ende der Hauptresorptionsphase werden sie dann wieder zurückgebildet.

Nach der Abbildung 9d von SCHNErder (1964) zu urteilen, kommen weniger differenzierte Mikrozotten auch in den Nahrungsvakuolen von Paramecium vor. SCHNEIDER hat diese Cytoplasmaprotuberanzen allerdings funktionell in einer anderen Weise interpretiert.

Für eine Resorption der Nahrung spricht ferner die äußere Deckschicht der Mikrozottenmembran, die ebenfalls am Dünndarmepithel auftritt (BERGENER 1962) und die nach experimentellen Beobachtungen an Amöben der initialen, selektiven Adsorption zu dienen scheint (vgl. Holter 1965, Komnick \& Wohlfarth-BotterMANN 1965). Im Gegensatz zu der pinocytotischen Stoffaufnahme bei Amöben, wo die Deckschicht während oder nach der Invagination der Pinocytoseblasen abgestoßen wird (Holter 1965), scheint ihre Ausbildung in den Nahrungsvakuolen von Metafolliculina mit der Entwicklung des Faltensaumes Hand in Hand zu gehen. In den jüngeren Nahrungsvakuolen ist allem Anschein nach zunächst nur eine diffuse Grundschicht vorhanden, während die härchenförmige Differenzierung erst bei den älteren Vakuolen zur deutlichen Ausprägung kommt.

Nach der zeitlich unterschiedlichen Aktivität lytischer Enzyme in den Nahrungsvakuolen haben Müller \& Törö (1962) zwischen Ingestions-, Digestions- und Egestionsvakuolen unterschieden. Nach unseren morphologischen Befunden erhebt sich die Frage, ob es nicht berechtigt ist, im "intrazellulären Verdauungstrakt" (DE DUvE 1963) von Metafolliculina darüber hinaus auch von einem Resorptionsvakuolenstadium zu sprechen, das bei diesem Vertreter einer phylogenetisch hoch differenzierten Ciliatengruppe allerdings strukturelle und funktionelle Parallelen eher zum Dünndarmepithel als zu den Lysosomen (vgl. Müller et al. 1963) aufweist. Es erscheint uns daher vorerst fraglich, ob es zweckmäßig ist, die Nahrungsvakuolen mehr oder weniger mit Lysosomen gleichzusetzen, wie dies in jüngster Zeit von den vorgenannten Autoren (vgl. Seite 213) versucht worden ist.

\section{ZUSAMMENFASSUNG}

1. Die Nahrungsvakuolen von Metafolliculina andrewsi entwickeln mit zunehmendem Alter einen Faltensaum, der seine höchste Differenzierung in dem Altersstadium erreicht, wo die stärkste Resorption der Nährstoffe zu vermuten ist.

2. Dieser Faltensaum besteht aus lamellenartigen Cytoplasmaprotuberanzen und be- 
wirkt - ähnlich wie der Bürstensaum an der freien Zelloberfläche - innerhalb der Zelle eine erhebliche Oberflächenvergrößerung der Nahrungsvakuole zugunsten der Permeation der Nährstoffe.

3. Bei alten Nahrungsvakuolen werden die Falten wieder zurückgebildet, eine Tatsache, die die funktionelle Bedeutung dieser Struktur weiterhin unterstreicht.

4. An Hand des Verdauungsgrades des Vakuoleninhalts und der morphologischen Veränderungen der Vakuolenwand lassen sich bei den Nahrungsvakuolen von Metafolliculina deutlich vier Alters- beziehungsweise Funktionsstadien unterscheiden: 1. Ingestionsvakuolen, 2. Digestionsvakuolen, 3. Resorptionsvakuolen und 4. Egestionsvakuolen.

5. Die morphologischen Befunde an der Resorptionsvakuole, die strukturelle und funktionelle Parallelen mit den resorbierenden Darmepithelien von Metazoen aufweist, werden im Rahmen der heutigen Vorstellungen über die zelluläre Stoffaufnahme diskutiert.

\section{ZITIERTE LITERATUR}

Andrews, E, A., 1923. Folliculina: case making, anatomy and transformation. J. Morph. 38, 207-278.

-- 1946. Ingestion organs in folliculinids and in stentor. J. Morph. 79, 419-444.

Bautzmann, H. \& Schmid, W., 1960. Vergleichende elektronenmikroskopische Untersuchungen am Amnion von Sauropsiden und Mammaliern (Huhn, Katze, Mensch), Z. Zellforsch. mikrosk. Anat. 51, 571-588.

BrRgener, M., 1962. Die Feinstruktur des Dünndarmepithels während der physiologischen Milchresorption beim jungen Goldhamster. Z. Zellforsch. mikrosk. Anat. 57, 428-474.

Brandt, P. W. \& Pappas, G. D., 1962. An electron microscopic study of pinocytosis in ameba. 2. The cytoplasmic uptake phase. J. biophys, biochem. Cytol. 15, 55-71.

Carasso, N., Fayard, P. \& Goldfischer, S., 1964. Localisation, à l'echelle des ultrastructures, d'activités de phosphatases en rapport avec les processus digestifs chez un cilié (Campanella umbellaria). J. Microscopie 3, 297-322.

Chapman-Andresen, C. \& Nilsson, J. R, 1960. Electron micrographs of pinocytosis channels in Amoeba proteus. Expl. Cell Res. 19, 631-633.

Duve, C. DE, 1963. The lysosome concept. In: Reudk, A.V.S. de \& M. P. Cameron (Eds): Lysosomes. Churchill, London, 1-35.

Favard, P. \& Carasso, N., I963. Mise en évidence d'un processus de micropinocytose interne au niveau des vacuoles digestives d'Epistylis anastatica (Cilié Péritriche). J. Microscopie 2, 495-498.

- 1964. Étude de la pinocytose au niveau des vacuoles digestives de ciliés péritriches. $J$. Microscopie 3, 671-696.

Gresson, R. A. R. \& Threadgold, L. T., 1959. A light and electron microscope study of the epithelial cells of the gut of Fasciola bepatica L. J. biopbys. biochem. Cytol. 6, 157-162.

Hadžı, J., 1951. Studien über Folliculiniden. Dela Slov. Acad. Znan. Umet. Hist. nat. Med. 4, 1-390.

Hayward, A. F., 1963. Electron microscopy of induced pinocytosis in Amoeba proteus. C. $r$. Trav. Lab. Carlsberg 33, 535-558.

Helander, H. F., 1962. Ultrastructure of fundus glands of the mouse gastric mucosa. J. Ultrastruct. Res. (Suppl.) 4, 1-123.

- 1965. Morphology of animal secretory gland cells. In: Wohlfarth-Bottermann, K. E. (Hrsg.): Sekretion und Exkretion. Springer, Berlin, 2-26.

Holter, H., 1965. Physiologie der Pinocytose bei Amöben. In: Wohlfarth-Bottermann, K. E. (Hrsg.): Sekretion und Exkretion. Springer, Berlin, 119-146. 
Jurand, A., 1961. An electron microscope study of food vacuoles in Paramecium aurelia. $J$. Protozool. 8, 125-130.

Koмnick, H., 1963. Zur funktionellen Morphologie der Salzsäureproduktion in der Magenschleimhaut. Histochemie 3, 354-378.

- 1965. Funktionelle Morphologie von Salzdrüsenzellen. In: Wohlfarth-Bottermann, K. E. (Hrsg.): Sekretion und Exkretion. Springer, Berlin, 289-314.

- \& Wohlfarth-Bottermann, K. E., 1964. Morphologie des Cytoplasmas. Fortschr. Zool. $17,1-154$.

-, - 1965. Das Grundplasma und die Plasmafilamente der Amöbe Chaos chaos nach enzymatischer Behandlung der Zellmembran. Z. Zellforsch. mikrosk. Anat. 66, 434-456.

Kurtz, S. M., 1964. The kidney. In: Kurtz, S. M. (Ed.): Electron microscopic anatomy. Academic pr., New York, 239-265.

Moericke, V. \& Wohlfarth-Bottermann, K. E., 1960. Zur funktionellen Morphologie der Speicheldrüsen von Homopteren. 4. Mitt. Die Ausführgänge der Speicheldrüsen von Myzus persicae (Sulz.), Aphididae. Z. Zellforsch. mikrosk. Anat. 53, 25-49.

Müller, M. \& RöHlich, P., 1961. Studies on feeding and digestion in Protozoa. 2. Food vacuole cycle in Tetrabymena corlissi. Acta morph. hung. 10, 297-305.

- \& Törö, I., 1962. Studies on feeding and digestion in Protozoa. 3. Acid phosphatase activity in food vacuoles of Paramecium multimicronucleatum. J. Protozool. 9, 98-102.

-, Röнlich, P., Tóth, J. \& Törö, I., 1963. Fine structure and enzymic activity of protozoan food vacuoles. In: Reuck, A. V. S. de \& M. P. Cameron (Eds): Lysosomes. Churchill, London, 201-225.

Palay, S. L. \& Karlin, L. J., 1959a. An electron microscopic study of the intestinal villus. 1. The fasting animal. J. biopbys. biochem. Cytol. 5, 363-372.

- 1959b. An electron microscopic study of the intestinal villus. 2. The pathway of fat absorption. J. biophys. biochem. Cytol. 5, 373-384.

Policard, A., Bessis, M., Collet, A. \& Giltaire-Ralyte, L., 1955. Une formation submicroscopique cellulaire peu connue: Les microvillosités. Bull. Microsc. appl. 5, 133-138.

Rhodrn, J., 1964. Correlation of ultrastructural organisation and function in normal and experimentally changed proximal convoluted tubule cells of the mouse kidney. Godvil, Stockholm.

- 1958. Anatomy of kidney tubules. Int. Rev. Cytol. 7, 485-534.

Roth, L. E, 1960. Electron microscopy of pinocytosis and food vacuoles in Pelomyxa. J. Protozool. 7, 176-185.

Ruska, C., 1960. Die Zellstrukturen des Dünndarmepithels in ihrer Abhängigkeit von der physikalisch-chemischen Beschaffenheit des Darminhaltes. 1. Wasser und Natriumchlorid. Z. Zellforsch. mikrosk. Anat. 52, 748-777.

SchmidT, W, 1965. Morphologische Aspekte der Stoffaufnahme und intrazellulären Stoffverarbeitung. In: Wohlfarth-Bottermann, K. E. (Hrsg.): Sekretion und Exkretion. Springer, Berlin, 147-161.

SCHNEIDER, L., 1964. Elektronenmikroskopische Untersuchungen an den Ernährungsorganellen von Paramecium. 2. Die Nahrungsvakuolen und die Cytopyge. Z. Zellforsch. mikrosk. Anat. 62, 225-245.

Sedar, A. W., 1964. Stomach and intestinal mucosa. In: Kurtz, S. M. (Ed.): Electron microscopic anatomy. Acad. pr., New York, 123-148.

SiTTE, H., 1965. Beziehungen zwischen Zellstruktur und Stofftransport in der Niere. In: Wohlfarth-Bottermann, K. E. (Hrsg.): Sekretion und Exkretion. Springer, Berlin, 343-377.

Smith, D. S. \& LitTAU, V. C., 1960. Cellular specialization in the excretory epithelia of an insect, Macrosteles fascifrons Stål. (Homoptera). J. biophys. biochem. Cytol. 8, 103-133.

Staubesand, J., 1965. Cytopempsis. In: Wohlfarth-Bottermann, K. E. (Hrsg.): Sekretion und Exkretion. Springer, Berlin, 162-189.

Steingrecht, R. A., 1964. Feinstruktur und Histochemie der Sexualduftdrüse des Seidenspinners Bombyx mori L. Z. Zellforsch. mikrosk. Anat. 64, 227-261.

- \& SCHneider, D., 1964. Die Faltung der äußeren Zellmembran in den SexuallockstoffDrüsenzellen des Seidenspinners. Naturwissenscbaften $\mathbf{5 1 , 4 1 ,}$ 
Thoenes, W., 1961. Die Mikromorphologie des Nephrons in ihrer Beziehung zur Funktion. T. 1. Funktionseinheit: Glomerulum - proximales und distales Konvolut. Klin. Wschr. 39, $504-518$.

- 1965. Transportwege in den Harnkanälchen der Säugerniere. In: Wohlfarth-Bottermann, K. E. (Hrsg.): Sekretion und Exkretion. Springer, Berlin, 315-342.

Uhlrg, G., 1963a. Untersuchungen über die Folliculiniden (Ciliata, Heterotricha) der Deutschen Bucht. Veröff. Inst. Meeresforsch., Bremerh. (Sonderbd.), 115-121.

- 1963b. Der Gehäusebau bei Metafolliculina andrewsi (Ciliata, Heterotricha). Zool. Anz. (Suppl. Bd.) 27, 498-507.

- Die mehrgliedrige Kultur litoraler Folliculiniden. Helgoländer wiss. Meeresunters. 12 , $52-60$.

Wohlfarth-Bottermann, K. E., 1957. Die Kontrastierung tierischer Zellen und Gewebe im Rahmen ihrer elektronenmikroskopischen Untersuchung an ultradünnen Schnitten. Naturwissenschaften 44, 287-288.

- 1960. Protistenstudien. 10. Licht- und elektronenmikroskopische Untersuchungen an der Amöbe Hyalodiscus simplex n. sp. Protoplasma 52, 58-107.

ZETTERQvist, H., 1956. The ultrastructural organization of the columnar absorbing cells of the mouse jejunum. Godvil, Stockholm. 